\title{
L'émergence de l'œuvre musicale et la construction conjointe de sa connaissance au cours des interactions didactiques
}

The emergence of the musical work and the joint construction of its knowledge in the didactic interactions

\section{Frédéric Maizières}

\section{OpenEdition Journals}

Édition électronique

URL : https://journals.openedition.org/educationdidactique/2473

DOI : 10.4000/educationdidactique.2473

ISSN : 2111-4838

\section{Éditeur}

Presses universitaires de Rennes

\section{Édition imprimée}

Date de publication : 30 mai 2016

Pagination : 77-96

ISBN : 978-2-7535-5060-5

ISSN : $1956-3485$

Référence électronique

Frédéric Maizières, « L'émergence de l'œuvre musicale et la construction conjointe de sa connaissance au cours des interactions didactiques », Éducation et didactique [En ligne], 10-1 | 2016, mis en ligne le 30 mai 2018, consulté le 23 août 2022. URL : http://journals.openedition.org/ educationdidactique/2473; DOI : https://doi.org/10.4000/educationdidactique.2473 


\title{
L'ÉMERGENCE DE L'GUVRE MUSICALE ET LA CONSTRUCTION CONJOINTE DE SA CONNAISSANCE AU COURS DES INTERACTIONS DIDACTIQUES
}

\author{
Frédéric Maizières \\ Maître de conférences en musicologie à l'ESPE de Toulouse \\ Chercheur en didactique des enseignements artistiques aux laboratoires \\ LLA-Créatis et UMR-EFTS de l'université de Toulouse - Jean Jaurès
}

\begin{abstract}
Lactivité d'écoute constitue la seconde pratique, avec l'interprétation vocale, de l'éducation musicale à l'école primaire en France, en vue d'acquérir la connaissance d'œuvres musicales. Une étude montre que certaines œuvres descriptives de la musique savante sont souvent étudiées, en revanche on ignore comment ces œuvres sont transposées dans la classe et quels dispositifs pédagogiques sont mis en place aujourd'hui par les enseignants généralistes. Quelle est la place de l'œuvre dans l'action de médiation ou d'enseignement? Quels savoirs sont en jeu et quelles relations intrinsèques entretiennent-ils avec l'œuvre?

Il s'agit, à partir d'une étude de cas, d'analyser le contenu des échanges didactiques articulés aux différentes écoutes de l'œuvre de Smetana, La Moldau, dans une classe de CM2, en vue de décrire et de comprendre comment la connaissance de l'œuvre musicale se co-construit au cours des interactions didactiques.
\end{abstract}

Mots-clés: éducation musicale, action didactique conjointe, interactions verbales, œuvre musicale, connaissance.

The emergence of the musical work and the joint construction of its knowledge in the didactic interactions

Along with the vocal performance the listening activity is the second practice of music education in primary school in France. It aims to have the children acquire the knowledge of musical works. A study shows that some descriptive works of art music are often studied, however it is unclear how these works are transfered over to the classroom and which teaching methods are developped today by generalist teachers. What is the place of the work in the mediation or teaching process? What knowledge is at stake and which inherent relations does it maintain with the work?

On the basis of a case study, we mean to analyze the content of educational exchanges articulated to the different listenings to the work of Smetana's The Moldau in a CM2 class (fifth grade), to describe and understand how the knowledge of the musical work is co-constructed in the didactic interactions.

Keywords: music education, didactic joint action, verbal interaction, musical work, knowledge. 
L'éducation musicale à l'école primaire s'appuie sur la pratique du chant et celle de l'écoute, dans l'objectif d'acquérir, entre autres, la connaissance d'œuvres musicales, en vue d'être " préparé à partager une culture européenne " (MENESR, 2006, p. 18 ; MEN, 2008a). En ce qui concerne l'activité vocale, on peut penser que la connaissance du chant est acquise lorsque les élèves ont les capacités de le restituer de mémoire en "respectant » la justesse mélodique, l'exactitude rythmique et celle des paroles, conformément au modèle donné initialement par l'enseignant ou avec un enregistrement (MEN, 2008a). Pour ce qui est de l'activité d'écoute, définir les savoirs, les capacités, les attitudes qui valident la connaissance d'une œuvre apparaît plus problématique.

Dans l'étude présentée ici, nous nous intéressons plus particulièrement à cette seconde activité qu'est l'écoute. Si les résultats d'une recherche antérieure montrent que les professeurs des écoles font majoritairement écouter de la musique savante (eux disent « classique »), notamment des œuvres descriptives ou dites « à programme " ou en lien avec un texte (opéras, contes musicaux) (Maizières, 2012, 2014), on ignore en revanche ce que deviennent ces œuvres dans la classe. À l'instar de Mili $(2007,2011)$ qui se pose la question des savoirs en jeu relativement aux nouvelles pratiques d'écoute en Suisse, on peut se demander comment émergent les savoirs sur l'œuvre musicale dans la classe et comment se co-construit sa connaissance en tant que savoir scolaire au cours des interactions entre l'œuvre (son enregistrement), l'enseignant et l'élève.

L'objectif de notre étude vise à décrire et à comprendre comment le savoir de référence (l'œuvre) est transposé dans la classe (savoir scolaire), sans intention prescriptive ou évaluative. À partir d'une situation d'enseignement, nous analysons plus particulièrement les interactions verbales qui s'articulent aux différents temps d'écoute, afin de mettre en évidence l'évolution de la représentation de l'œuvre au cours de la situation. L'étude s'appuie sur une séance ordinaire, c'est-à-dire conçue et mise en œuvre par un professeur des écoles (PE dans la suite du texte) selon son habitude et sa programmation personnelle sans aucune intervention du chercheur.

Dans une première partie, nous présentons quelques aspects de la didactique de l'écoute musicale à l'école, puis nous montrons comment la théorie de l'action conjointe en didactique nous permet d'accéder à l'intelligibilité de la pratique enseignante en mettant notamment au jour les dynamiques évolutives du savoir enseigné et appris au fil des interactions (Amade-Escot, 2014 ; Amade-Escot \& Venturini, 2009 ; Sensevy, 2007, 2008, 2011 ; Venturini \& Tiberghien, 2012). Dans une seconde partie, nous présentons le contexte de l'étude et les outils méthodologiques. Dans une troisième partie, nous décrivons la situation d'enseignement-apprentissage concernant l'étude de La Moldau de Smetana dans une classe de $\mathrm{CM} 2$ à partir des différentes formes d'émergence du savoir, soit le savoir transmis par l'enseignant, les écoutes de l'enregistrement et le savoir co-construit au cours des interactions verbales. Dans une quatrième partie, nous analysons l'action conjointe des élèves et du professeur dans l'émergence des caractéristiques de l'œuvre en portant notre attention sur la triple dimension mésogénétique, topogénétique et chronogénétique de l'activité.

\section{CADRE THÉORIQUE : DIDACTIQUE DE L'ÉCOUTE MUSICALE ET THÉORIE DE L'ACTION CONJOINTE EN DIDACTIQUE (TACD)}

\section{L'écoute musicale à l'école primaire}

Dès la maternelle, les activités d'écoute musicale sont envisagées dans l'objectif d'affiner « l'attention », de développer « la sensibilité, la discrimination des sons et la mémoire auditive » (MEN, 2008a, p. 16). Les objectifs sont liés à la fois aux domaines de la cognition et à ceux de la sensibilité. À travers les compétences visées, on perçoit une démarche qui, sans être prescrite avec précision, est très largement fondée sur les échanges verbaux, puisqu'il s'agit pour l'élève, dès la fin de la maternelle, d'être capable d'" écouter un extrait musical ou une production, puis [de] s'exprimer et dialoguer avec les autres pour donner ses impressions » (MEN, 2008a, p. 16). Si le plaisir de l'écoute est clairement annoncé dans les programmes de maternelle, cet objectif est rejoint au cycle 2 par l'approche des œuvres à partir d'éléments mélodiques, rythmiques, dynamiques et relatifs aux timbres, puisqu'il s'agit pour l'élève d'être capable de repérer les éléments caractéristiques d'une ouvre (MEN, 2008a, p. 19). Enfin, au cycle 3, le travail de perception et d'identification des éléments caractéristiques d'une œuvre est prolongé par la découverte des genres et des styles musicaux divers. En conclusion, les prescriptions relatives à l'écoute d'œuvres musi- 
cales, en lien direct avec l'enseignement de l'histoire des arts, visent à faire acquérir à l'élève une culture par la connaissance d'un certain nombre d'œuvres et la capacité de les reconnaître, de les comparer et de les situer dans le temps et dans l'espace (MENESR, 2006 ; MEN, 2008a, 2008b).

Il semble important de relever que les programmes, sans fixer les œuvres que les enseignants doivent faire connaître à leurs élèves, ni la démarche d'étude qu'ils doivent mettre en place, précisent un certain nombre de points importants nécessaires pour l'analyse et la compréhension de la situation d'enseignement-apprentissage de l'écoute musicale. Celle-ci s'appuie sur des œuvres variées puisqu'il s'agit d'amener l'élève à acquérir la connaissance des principaux genres et styles musicaux. Nous avons également pointé l'importance des échanges verbaux qui vont précéder ou succéder les différentes écoutes, partielles ou totales, de l'œuvre au cours d'une même séance. Il convient enfin de souligner l'articulation entre les émotions et les préférences de l'élève, et les connaissances présentes et à construire (MEN, 2008a, p. 28). Le savoir est construit essentiellement à partir de ce que l'élève perçoit, identifie mais aussi ressent et plus rarement à partir d'informations théoriques données par l'enseignant ou recherchées par l'élève (Terrien, 2006 ; Mili, 2011). On peut, à l'instar de Mili, parler de "maïeutique de l'écoute». "L'accoucheur étant l'enseignant, bien sûr, mais avec des élèves très actifs dans le processus, puisqu'ils sont conviés à faire écho, avec leurs mots, aux extraits d'œuvres écoutées, pour que les notions puissent émerger et les apprentissages se faire. "(Mili, 2011, p. 155.) Il s'agit pour l'enseignant « d'articuler approches externes et internes des ouvres musicales » et de « situer son intervention à leur intersection », non seulement comme le précise Mialaret, pour pouvoir comprendre et évaluer les obstacles qui pourraient empêcher les élèves d'accéder à une œuvre, mais aussi comme un moyen d'amener l'élève à atteindre les caractéristiques esthétiques de l'œuvre et à faire évoluer sa représentation (Mialaret, 2001, p. 109). Ces caractéristiques sont exprimées par les élèves à travers la parole, mais aussi par d'autres moyens en complémentarité ou en substitution, comme par exemple, l'expression corporelle libre, l'imitation vocale ou instrumentale, la représentation graphique, la création d'une séquence sonore. Mais quel que soit le moyen utilisé pour amener l'élève à s'exprimer sur l'œuvre, on peut faire le postulat, à l'instar de Grandaty (2005), que dans la situation d'écoute musicale, l'oral « fonctionne comme un opérateur d'apprentissage ». Ainsi, comme le rappelle l'auteur, « focaliser l'étude des relations entre l'activité verbale et l'apprentissage conduit à accorder une importance particulière à la notion de tâche » (Grandaty, 2005, p. 51), l'amenant à distinguer deux types de tâches, la tâche disciplinaire et la tâche langagière. Dans la situation d'écoute musicale, deux types de tâches alternent, il s'agit des écoutes de l'enregistrement de l'œuvre et des échanges verbaux qui en découlent ou qui les initient. En effet, les différentes écoutes sont articulées à des échanges au cours desquels l'enseignant donne les consignes et les informations qu'il juge nécessaires et les élèves s'expriment sur ce qu'ils ont entendu (identification des éléments perçus, expression de leurs émotions, de leur préférence puis mise en relation de ces éléments). Grandaty caractérise la tâche par « un résultat, un but à atteindre, des contraintes dans l'obtention de ce résultat, et un domaine de connaissances spécifiques » (p. 51). Ainsi la tâche qui consiste à écouter une œuvre musicale vise l'élaboration d'une représentation de cette œuvre à partir de laquelle peuvent s'organiser les échanges au sein de la classe. Une des principales contraintes tient à la confrontation de perceptions différentes et à la prise en compte des individualités dans le « collectif didactique » (Sensevy \& Mercier, 2007, p. 194). Grandaty souligne encore qu'une tâche correctement prescrite ne débouche pas obligatoirement sur une activité d'apprentissage. La tâche qui consiste pour l'élève à écouter une œuvre musicale en est un exemple particulièrement illustrant. Quelle que soit l'attitude de l'élève au cours de cette tâche, il n'est pas certain que s'élabore une représentation spécifique de l'œuvre. L'élève peut tout à fait simuler une posture d'écoute " pour faire bonne figure et marquer sa bonne volonté » sans effectuer réellement le travail et laisser aller son imagination vers d'autres pensées empêchant toute perception de la musique. D'où la nécessité d'élaborer collectivement des conduites discursives, afin de permettre à l'élève de trouver « un étayage collectif et une incitation à construire une réponse qui est assumée par plusieurs élèves » (Grandaty, p. 60). Conséquemment, l'analyse de ces conduites verbales représente un accès à ce que les élèves sont en train de construire, de comprendre et d'apprendre (Chabanne, Parayre, Villagordo \& Dequin, 2011). 
Action (conjointe), transaction (didactique), interaction (verbale)

Notre étude vise à analyser une situation d'enseignement-apprentissage qui s'appuie essentiellement sur des interactions langagières, entre un enseignant qui enseigne l'écoute d'une œuvre musicale et des élèves censés en acquérir une représentation en tant que connaissance spécifique inter et intra-individuelle. La situation d'enseignement-apprentissage d'une œuvre peut être vue comme une " action didactique conjointe $»$, soit une action produite par des individus « au sein d'une relation ternaire entre le savoir, le professeur, et les élèves " " dans des lieux (institutions) où l'on enseigne et où l'on apprend » (Sensevy, 2007, p. 14-15). Sensevy (2007) insiste sur le caractère coopératif de l'action didactique conjointe en ce sens que l'on mesure les effets de l'action de l'enseignant sur le savoir des élèves et que l'action enseignante évolue en fonction de la réaction des élèves. Il considère les interactions didactiques comme des «transactions », entendues comme des actions dialogiques, conjointes et organiquement coopératives où enseignants et élèves sont co-acteurs et co-locuteurs (Sensevy, 2007, 2008, 2011). La transaction se situe dans un environnement institutionnel particulier (ici une classe de CM2) à propos d'un objet particulier (ici l'œuvre La Moldau de Smetana).

Pour ces raisons, Sensevy $(2007,2011)$ voit dans le modèle du jeu un moyen de décrire et de comprendre l'action didactique, qu'il considère comme une succession de jeux (didactiques). Chaque jeu comporte des joueurs représentant respectivement l'instance professeur (P) et l'instance élève (E) (Sensevy, 2007, p. 14). E gagne s'il produit la stratégie gagnante de son propre mouvement (proprio motu). P, qui connaît la stratégie gagnante, gagne si E gagne. Il s'agit donc d'un jeu collaboratif, un jeu conjoint inclus dans une action conjointe (Sensevy, 2008, p. 44). Toutefois, l'action conjointe ne signifie pas pour autant que $\mathrm{E}$ et $\mathrm{P}$ aient les mêmes buts, si bien que des transactions à propos du savoir concerné sont inévitables au cours du jeu pour que celui-ci progresse.

Pour gagner à ce jeu, le professeur va engager les élèves dans une succession de jeux d'apprentissages, qui sont déterminés par un « contrat » et par la confrontation des élèves à un certain « milieu » (Brousseau, 1998 ; Sensevy \& Mercier, 2007). Le processus didactique suppose la création d'un milieu défini par Brousseau (1998) comme «tout ce avec quoi l'élève se trouve en relation au moment de l'apprentissage », qu'ils s'agissent d'éléments matériels (l'enregistrement de l'œuvre, un texte, une partition...) ou symboliques et cognitifs (notions déjà connues, démarches didactiques connues, etc.). Le milieu correspond donc aux éléments matériels et symboliques qui vont nourrir l'action de l'élève, ou résister à son action. Le contrat correspond aux attentes réciproques du professeur et des élèves, « système d'attentes constitué dans ses dimensions tant génériques que spécifiques au savoir visé, sur le fond des habitudes de la classe, du professeur et des élèves dans des situations antérieures » (Forest \& Batézat-Batellier, 2013, p. 80).

Pour initier et maintenir la relation didactique au cours du jeu, le professeur intervient de différentes manières et un quadruplet de descripteurs du jeu didactique en rend compte : définir, dévoluer, réguler, institutionnaliser (Sensevy, 2007, p. 28). Ainsi, le professeur définit le jeu didactique en introduisant les objets nécessaires et en précisant ses règles. Il dévolue le jeu, en agissant de telle manière que les élèves acceptent de s'engager dans le jeu et d'y jouer de manière adéquate. Il s'agit là d'un processus qui se poursuit tout au long du jeu. Il régule aussi le jeu, en intervenant pour que les élèves modifient leur action et parviennent à gagner. Enfin, il institutionnalise le savoir en jeu, signifiant aux élèves qu'ils ont produit le savoir enjeu du jeu et que celui-ci est valide au-delà de la classe, dans une communauté beaucoup plus large.

Nos travaux s'inscrivent délibérément dans une approche considérant que la diffusion des savoirs est au cœur de l'activité enseignante, y compris et plus encore, dans les disciplines artistiques. Si le savoir « à enseigner », soit l'œuvre à étudier, est choisi et amené par l'enseignant, nous faisons l'hypothèse que le savoir " enseigné » et " assimilé » se co-construit au cours des interactions didactiques. Toutefois, nous ne pouvons observer du côté des élèves que les signes qu'ils manifestent, notamment les propos qu'ils expriment au sujet de l'œuvre, expression guidée dans un milieu fortement organisé par l'enseignant. Ainsi, l'analyse des interactions verbales se focalisera plus particulièrement sur les trois dynamiques (les genèses), du milieu ( «mésogenèse »), du temps didactique ( " chronogenèse ») et des places et des responsabilités de chacun («topogenèse ») 
sous l'effet de l'action conjointe professeur-élèves (Sensevy \& Mercier, 2007). Nous avons vu que dans le processus didactique, les objets de savoir, relatifs à une organisation de connaissances, forment un milieu. "La mésogenèse décrit le processus par lequel le professeur et les élèves organisent ou réorganisent le milieu » (Venturini \& Tiberghien, 2012, p. 99). Le processus didactique se caractérise également par un savoir en évolution constante. La chronogenèse concerne l'avancée temporelle des savoirs dans le système didactique. Enfin, le processus didactique suppose des acteurs, dont les positions ne sont pas équivalentes. La topogenèse permet de considérer la répartition des responsabilités épistémiques entre l'enseignant et les élèves dans les transactions didactiques. Les différents auteurs s'accordent sur la relation étroite entre ces trois dynamiques qui « évoluent de concert » dans la situation didactique (Schubauer-Leoni, 2008 ; Amade-Escot \& Venturini, 2009). Quelles dynamiques mésogénétiques, chronogénétiques et topogénétiques peut-on voir à l'œuvre dans une situation d'écoute musicale?

\section{MÉTHODOLOGIE}

Un double point de vue sur la pratique réelle : vidéo et autoconfrontation

Les données à disposition sont issues de plusieurs pièces :

- la vidéo d'une séance de classe : d'une durée de 80 minutes, elle a été réalisée dans une classe de CM2 et concerne l'étude de l'œuvre de Smetana, La Moldau;

- un entretien post-séance réalisé immédiatement après la séance au cours duquel l'enseignant explique ses objectifs, sa démarche, le choix des outils ;

- un entretien d'autoconfrontation réalisé plusieurs semaines après la séance, dans le but d'éclairer le chercheur sur « les points aveugles de l'activité ». En visionnant sa propre pratique, le sujet observé s'exprime sur ce qu'il réalise et le contexte de cette action en réaction spontanée ou en réponse aux questions du chercheur. Une situation d'enseignement-apprentissage réelle, c'est-à-dire en dehors de l'expérience, s'inscrit dans une progression et n'existe jamais seule. Sa compréhension nécessite de s'appuyer aussi sur ce qui la précède et ce qui suivra (Leblanc, Ria \&
Veyrunes, 2013). L'entretien d'autoconfrontation représente aussi pour nous le moyen de confronter notre analyse à celle du sujet observé. Les données ainsi obtenues incitent à une analyse qui évite le " court-circuitage de l'implicite évaluatif » (Bain, 2002, p. 331) que pourrait constituer notre propre expérience de la formation en éducation musicale.

\section{Contextualisation de l'œuvre et de la séance}

La situation observée concerne l'étude de $L a$ Moldau de Smetana ${ }^{1}$. L'objectif de la séance vise à « faire ressortir les différents éléments que voulait faire apparaître le compositeur » (entretien post). Cet objectif déclaré par l'enseignant est en adéquation avec les programmes qui mentionnent le repérage des éléments caractéristiques d'une œuvre en vue d'en construire sa connaissance (MEN, 2006, 2008a). L'œuvre composée en 1874 appartient au genre « poème symphonique »; elle est donc considérée comme une œuvre dite "à programme ». Le genre « musique à programme » désigne la « musique d'essence narrative, évocatrice, descriptive ou illustrative, donc renvoyant à une donnée extramusicale; cela par opposition à la musique pure, qui ne ferait appel qu'à une perception abstraite, sans référence à aucun élément extramusical » (Vignal, 1987 / 2005, p. 682). Dans la situation observée, l'œuvre est présente par l'intermédiaire de son enregistrement diffusé à partir d'un ordinateur portable relié à un Tableau Numérique Interactif (TNI) «pour la qualité du son », précise l'enseignant (entretien d'autoconfrontation). La qualité de diffusion est effectivement satisfaisante et permet à tous les élèves d'entrer dans la tâche d'écoute que l'enseignant reproduit à 4 reprises. L'étude de cette ouvre est organisée sur une seule séance, mais on apprend au cours de celleci et de l'entretien post que la classe y reviendra pour y traiter la question des instruments. Au cours de l'entretien d'autoconfrontation l'enseignant reconnait que la séance est longue et déclare qu'en dehors du contexte de l'observation filmée, il l'aurait sans doute menée en deux temps, soit deux fois 40 minutes: « j'aurais laissé une affiche... reprenant les éléments que j'aurais mis comme je fais souvent sur ce tableaulà... voilà en attente quoi ».

La place de l'œuvre est définie par des paramètres chronologiques. D'une part, l'œuvre dure 12 minutes et est diffusée 4 fois en totalité. D'autre part, la séance 
se compose d'une alternance d'interactions entre l'œuvre et les élèves et d'interactions langagières entre les élèves et l'enseignant. L'objectif est d'amener les élèves à identifier les différents tableaux évoqués par la musique. L'enseignant juge que l'objectif est très accessible dans le cadre de cette oeuvre qu'il a déjà étudiée antérieurement avec d'autres classes (entretien post). Chacune des auditions de l'œuvre est dépendante du type de tâches demandées aux élèves (recueillir les impressions générales, identifier les différentes parties, comprendre la chronologie, illustrer).

\section{L'ÉMERGENCE DES SAVOIRS SUR L'CEUVRE DANS LA CLASSE}

La description de la situation d'enseignementapprentissage observée devrait nous permettre de montrer comment l'œuvre musicale et ses caractéristiques, en tant que savoirs, émergent dans la classe. La description se focalise sur trois formes d'apparition du savoir : la transmission de connaissances par l'enseignant, les différentes écoutes de l'œuvre et les interactions verbales entre les élèves et le PE. La mise en évidence de ces trois formes d'émergence des savoirs nous amène à un premier niveau d'analyse, celui des jeux d'apprentissage. Au niveau du jeu didactique, le $\mathrm{PE}$ cherche à faire acquérir aux élèves une représentation de l'œuvre, notamment de son programme et de son organisation, à partir des écoutes successives et de la confrontation des éléments caractéristiques perçus. Un deuxième niveau d'analyse nous amène à spécifier le jeu didactique en une succession de jeux d'apprentissage, dont le tableau suivant présente une typologie (tableau 1).

Tableau 1. Synopsis de la séance (temps en minutes)

\begin{tabular}{|c|c|}
\hline 0:00 & $\begin{array}{l}\text { Jeu } 1 \text { : première consigne d'écoute. } \\
\text { Le PE rappelle les conditions matérielles d'écoute et introduit brièvement l'œuvre. Un élève questionne } \\
\text { sur le sens du terme symphonique. Au cours de la réponse, le PE donne des précisions sur le contexte } \\
\text { historique de l'œuvre et termine en donnant un «indice » d'écoute. }\end{array}$ \\
\hline $2: 47$ & $\begin{array}{l}\text { Jeu } 2 \text { : première écoute de l'œuvre en entier. } \\
\text { Élèves et PE écoutent sans rien dire. }\end{array}$ \\
\hline $14: 43$ & $\begin{array}{l}\text { Jeu } 3 \text { : parler sur l'œuvre. } \\
\text { Les élèves disent ce qu'ils ont perçu et identifié. Les propos des élèves répondent directement à l'indice et } \\
\text { se centrent sur l'élément naturel qu'est censée évoquer l'œuvre. Le PE distribue une carte géographique } \\
\text { sur laquelle les élèves peuvent repérer La Moldau. }\end{array}$ \\
\hline $15: 43$ & $\begin{array}{l}\text { Jeu } 4 \text { : deuxième consigne d'écoute qui consiste à identifier le nombre de parties. } \\
\text { Le PE oriente et définit rapidement la seconde écoute vers le repérage des différentes parties. }\end{array}$ \\
\hline $21: 50$ & $\begin{array}{l}\text { Jeu } 5 \text { : deuxième écoute de l'œuvre en vue d'identifier le nombre de parties. } \\
\text { Les élèves sont réunis en binôme pour compter le nombre de parties de l'œuvre. Ils sont censés commu- } \\
\text { niquer entre eux, mais peu le font et l'écoute est majoritairement individuelle. }\end{array}$ \\
\hline $33: 47$ & $\begin{array}{l}\text { Jeu } 6 \text { : dire et justifier le nombre de parties identifiées. } \\
\text { Les élèves parlent des variations d'intensité, d'orchestration. Le PE oriente le questionnement sur le } \\
\text { thème musical en vue de préparer la troisième écoute. }\end{array}$ \\
\hline $37: 27$ & $\begin{array}{l}\text { Jeu } 7 \text { : troisième écoute (temps indiqués) fractionnée et commentée de l'œuvre. } \\
\text { Les élèves doivent interrompre la musique pour indiquer les changements de parties et justifier. Devant } \\
\text { la difficulté des élèves, le PE reprend la main à certains moments et stoppe lui-même l'écoute en deman- } \\
\text { dant aux élèves de justifier. À d'autres moments, les commentaires sont simultanés à l'écoute de l'enre- } \\
\text { gistrement (temps soulignés) : de } 37: 58 \text { à } 38: 57 \text {, de } 40: 19 \text { à 44:08, de } 44: 40 \text { à } 45: 00 \text {, de 45:46 à } 46: 36 \text {, } \\
\text { de } 47: 35 \text { à 49:00, de 49:24 à 52:23, de 53:01 à 54:59, de 55:47 à 56:33, de 56:58 à 57:42, de 57:58 } \\
\text { à 58:16 et de 59:21 à 59:25. }\end{array}$ \\
\hline $59: 35$ & $\begin{array}{l}\text { Jeu } 8 \text { : lire et comprendre le poème. } \\
\text { Un élève lit le poème sur lequel s'est appuyé Smetana pour composer l'œuvre. Le PE commente. }\end{array}$ \\
\hline $64: 20$ & $\begin{array}{l}\text { Jeu } 9 \text { : compléter le tableau par un titre pour chacune des parties. } \\
\text { Les élèves ont un tableau de dix lignes qu'ils doivent compléter par un titre correspondant à chacune des } \\
\text { dix parties de l'œuvre à l'aide du poème et des photos des environnements évoqués par la musique que } \\
\text { le PE projette à l'écran. Un élève réclame une quatrième écoute de l'œuvre. }\end{array}$ \\
\hline $76: 53$ & $\begin{array}{l}\text { Jeu } 10 \text { : confronter ses réponses avec la correction donnée par le PE (tableau complété). } \\
\text { Les élèves confrontent leurs réponses avec le tableau proposé par le PE. }\end{array}$ \\
\hline
\end{tabular}


Le tableau 1 présente successivement les différents jeux tels que nous les avons découpés en vue de repérer les différentes « scènes » de l'action (Sensevy, 2007). Leur dénomination correspond à l'activité que les élèves sont censés réussir pour " gagner le jeu » correspondant. Elle est fondée sur le sens donné au jeu par l'enseignant, qui peut être différent de celui donné par les élèves. Le découpage en jeux résulte d'une analyse des enjeux de savoir dans la situation et des changements importants dans le milieu et le contrat didactique.

\section{Le savoir transmis par l'enseignant}

\section{Le contexte de l'ouvre : élément de définition du jeu}

Élément important de la séance, le discours introductif (jeu 1) concerne « ce qui se dit avant le dévoilement de l'œuvre, avant sa présentation matérielle » (Chabanne \& Dufays, 2011, p. 12). Dans la séance observée, le discours introductif distingue les deux fonctions décrites par Chabanne et Dufays (2011): la fonction « informative » (donner les règles, donner des connaissances) et la fonction "apéritive » (donner envie). Ainsi, le contrat vise l'écoute attentive de l'œuvre en l'absence d'information afin de laisser l'élève dans l'attente de toute représentation pour écouter (extrait 1).

Pour le PE, les conditions favorables à l'écoute d'une œuvre nécessitent une disponibilité physique et intellectuelle (faire « un peu le vide pour pouvoir être plus disponible ") à laquelle il se dit attaché (« oui, c'est très important », autoconfrontation). La courte présentation de l'œuvre qu'il fait sur son genre et son style répond à la volonté de ne pas « écraser d'avance les singularités des réceptions individuelles » (Chabanne \& Dufays, 2011, p. 13) et de susciter la curiosité de l'élève en limitant les informations : « je ne vous en dis pas plus» (min 0:35).

Mais, pour répondre à la question d'un élève sur le sens du mot « symphonique » (min 0:57), l'enseignant développe d'autres informations sur l'œuvre, notamment les intentions de l'auteur et le contexte, contrairement ce qu'il souhaitait au départ (réti-

Tableau 2. Extrait 1 de la situation, de min 0:00 à min 2:47

\begin{tabular}{|c|c|c|}
\hline 0:00 & PE & $\begin{array}{l}\text { (PE circule dans la classe) on pousse les trousses vous n'avez pas besoin des trousses vous } \\
\text { n'avez pas besoin de machine à calculer de : c'est bon tout va bien XXXX / on a juste besoin } \\
\text { de ses oreilles / d'accord : (pousse la table d'un élève pour aller à son bureau) pardon tu peux } \\
\text { pousser un peu ta table: voilà }\end{array}$ \\
\hline $0: 35$ & $\mathrm{PE}$ & $\begin{array}{l}\text { Alors justement pour l'instant / vous allez écouter / vous allez écouter une œeuvre classique } \\
\text { qui fait partie d'un poème d'un poème symphonique / d'accord / un poème symphonique qui } \\
\text { a donc six parties, donc six plans (geste de la main pour montrer qu'il ne veut pas en dire plus) / } \\
\text { je ne vous en dis pas plus pour le moment }\end{array}$ \\
\hline $0: 57$ & E1 & Ça veut dire quoi symphonique? \\
\hline $1: 00$ & $\mathrm{PE}$ & $\begin{array}{l}\text { Symphonique / qui fait partie d'une symphonie / qui est joué par un orchestre (geste circulaire } \\
\text { avec les mains pour montrer un ensemble) d'accord / bien / heu : alors cette œeuvre donc elle fait } \\
\text { partie donc : de : de ce poème symphonique qui s'appelle ma patrie / qui a été composé par } \\
\text { un musicien tchèque qui s'appelle Smetana / Bedrich Smetana / et qui avait composé cette } \\
\text { œuvre car son pays la Tchéquie était sous le joug autrichien en } 1800 \text { dans les années } 1800 \text { : } \\
\text { d'accord pour à peu près situer / d'accord / et / les tchèques s'étaient un petit peu révoltés } \\
\text { contre les : les envahisseurs autrichiens et : et ils ont réussi à se libérer des autrichiens / et } \\
\text { donc cette œuvre c'était pour : pour exalter / d'accord / le sentiment national / tout ce qui } \\
\text { était tchèque : typiquement tchèque et cetera / d'accord / bien / alors / heu : c'est à la gloire } \\
\text { de son pays / d'accord / cette œeuvre / donc dans cette œeuvre vous allez découvrir / mais il } \\
\text { parle d'un élément / de son pays / / et cet élément c'est un élément naturel / voilà pour } \\
\text { l'instant c'est le seul petit indice que je vous donne (geste de la main pour montrer la } \\
\text { restriction) alors écoutez bien / (s'adresse à un élève) ne bricole pas c'est pas le moment } \\
\text { non non / c'est pas le moment de réparer le compas: chut / c'est bon on y va (se dirige vers } \\
\text { l'ordinateur) on écoute }\end{array}$ \\
\hline $2: 47$ & & PE Démarre l'écoute \\
\hline
\end{tabular}

PE désigne l'enseignant, E désigne un élève, Es désigne un ensemble d'élèves. Les propos soulignés en gras sont ceux sur lesquels portera plus particulièrement l'analyse. 
cence). Il termine son propos en donnant « un petit indice » (min 2:20). La fonction de l'indice n'est-elle pas de favoriser l'action de l'élève ? Cet exemple illustre la dialectique entre réticence et expression dans laquelle est pris le PE (Sensevy, 2007, 2008). Celui-ci fait volontairement de la « rétention d'information ", de la "réticence ", en vue de favoriser «l'expression» du savoir par l'élève (Sensevy, 2007). Pour ce faire, il produit un signe (« c'est le seul petit indice que je vous donne $»$ ).

Si d'un point de vue de la mésogenèse, l'enseignant introduit des éléments matériels nouveaux, suite à la question de l'élève (min 1:00), ces éléments ne modifient pas fondamentalement l'intention de départ de ne pas dévoiler l'œuvre (réticence). En effet, les informations données sur le contexte historique et l'origine de l'œuvre ne permettent pas, nous semble-t-il, de dévoiler à un élève de 9-10 ans ce qu'il va entendre par la suite, comme le prévoyait le contrat initial.

\section{Les informations non verbales : des éléments de régulation et / ou d'institutionnalisation}

Les propos visant à contextualiser l'œuvre ne se limitent pas au discours introductif. C'est encore une fois pour répondre à la question d'un élève (min 17:58) que l'enseignant apporte d'autres infor- mations sur l'œuvre, notamment après les premiers échanges succédant à la première écoute (extrait 2). La fonction informative de ces propos a pour but de valider les réponses des élèves et de les guider dans leur analyse de l'œuvre (« vous avez bien trouvé de vous-mêmes [...] on va donc regarder le nombre de parties », min 18:21), tout comme les différents supports sur lesquels l'enseignant s'appuie tout au long de la séance (carte, texte, tableau, photographies).

En dehors des informations verbales, l'enseignant s'appuie au cours de la séance sur quatre types de supports dans le but d'apporter des informations supplémentaires sur le contexte et le sens de l'œuvre, mais aussi de valider et de formaliser le savoir. Il s'agit, tout d'abord d'une carte géographique qui montre l'existence de la rivière décrite par l'œuvre de Smetana (extrait 2). On peut penser que l'enseignant vise ici l'institutionnalisation du savoir mis en évidence par les élèves, néanmoins ces informations semblent vouloir davantage encourager ces derniers dans leurs propositions, plutôt que fixer un savoir définitif et transférable. En effet, les propos de l'enseignant sur le caractère réel, concret de l'élément extra-musical repéré (la rivière) n'apparaît pas comme un savoir lié à cet élément, mais davantage comme la volonté de valider ce qui a été perçu et dit par les élèves : " voyez je ne vous raconte pas d'histoire / c'est une vraie rivière hein / La Moldau / c'est

Tableau 3. Extrait 2 de la situation, de min 17:58 à min 19:06

\begin{tabular}{|c|c|c|}
\hline $17: 58$ & E10 & La bohème? \\
\hline \multirow[t]{5}{*}{$\begin{array}{l}18: 07 \\
18: 21\end{array}$} & PE & $\begin{array}{l}\text { (poursuit la distribution du document) Oui la Bohème c'est une région la Bohème / et donc / voyez } \\
\text { qui existe hein / d'accord / vous le regarderez tout à l'heure / simplement je vous montre / l'aspect } \\
\text { géographique de l'œuvre / d'accord / donc on parle d'un / élément existant / une rivière qui } \\
\text { existe / qui existe encore de nos jours forcément d'accord / et / le compositeur a réussi à vous } \\
\text { faire passer vous voyez bien je ne vous ai rien dit au début vous avez bien trouvé de vous- } \\
\text { mêmes / bon alors c'est rigolo parce que certains m'ont parlé de forêt / et on verra tout à l'heure } \\
\text { qu'il y a aussi la forêt / à un moment donné cette rivière elle va traverser une forêt / on va donc } \\
\text { regarder le nombre de : de parties / de cette œuvre / d'accord }\end{array}$ \\
\hline & & \\
\hline & E10 & XXXX \\
\hline & $\mathrm{PE}$ & Oui c'est en république tchèque oui oui c'est en république tchèque \\
\hline & E11 & XXXX \\
\hline $18: 53$ & PE & $\begin{array}{l}\text { Ah non non c'est en République tchèque / bien alors maintenant vous voyez vous avez vu votre } \\
\text { feuille / vous avez vu je ne vous raconte pas d'histoire hein / c'est une vraie rivière hein / La } \\
\text { Moldau / c'est pas une rivière imaginaire / que le compositeur a voulu / vous : décrire à travers } \\
\text { sa musique / d'accord / alors maintenant vous mettez cette feuille dans votre casier / (les élèves } \\
\text { rangent la feuille) / vous la regarderez un peu plus tard / et on va réécouter l'œeuvre / oui (un élève } \\
\text { lève le doigt) }\end{array}$ \\
\hline
\end{tabular}


pas une rivière imaginaire...» (min 18:53). On peut voir dans les propos insistants de l'enseignant pour reconnaître la "stratégie gagnante », l'indice d'un « effet Jourdain » (Brousseau, 1998) : « vous voyez bien je ne vous ai rien dit au début vous avez bien trouvé de vous-mêmes» (min 18:21).

Au cours de la quatrième écoute de l'œuvre, l'enseignant projette des photographies sélectionnées sur Internet pour illustrer les différents environnements évoqués par la musique de Smetana (jeu 9). Là encore aucune connaissance du contexte géographique ou humain n'est recherchée. La projection vise ici à aider les élèves dans la mise en évidence des dix parties de l'œuvre (« vous vous aidez des photos pour remplir votre tableau », min 66:07). Bien que leur caractère authentique soit encore souligné par le PE ( « qui sont d'ailleurs les vraies photos de $\mathrm{La}$ Moldau », min 65:10), les photos projetées sur le TNI ont davantage une fonction illustrative et constitue une manière pour l'enseignant de réguler le jeu qui consiste à trouver un titre à chacune des parties.

Enfin, on peut voir une volonté d'institutionnalisation avec l'étude du texte qui serait à l'origine de la composition et le tableau résumant chacune des dix parties de l'œuvre par un titre et destiné à valider le travail des élèves (jeu 10). Le PE explique aux élèves que pour composer La Moldau, Smetana s'est inspiré d'un texte: " je vais vous donner un petit texte / vous allez le lire / c'est à partir de ce texte que Smetana a écrit ce passage » (min 59:35). À l'issue de la troisième écoute, la lecture du texte par un élève et les commentaires de l'enseignant permettent à la classe de vérifier le déroulement chronologique de l'œuvre mis en évidence au cours de la séance. La trace écrite finale consiste à compléter un tableau de dix lignes par un titre correspondant à chacune des dix parties en s'appuyant sur le texte et avec l'aide des photos projetées et de l'enregistrement (jeu 9). La formalisation du savoir prend sa forme définitive avec le tableau récapitulatif distribué en fin de séance et censé permettre aux élèves de vérifier leurs réponses : "Vous pouvez retourner la feuille que je vous ai distribué : vous avez les dix parties vous avez vu / donc première source deuxième source... » (min 77:20).

\section{L'écoute de l'enregistrement de l'œuvre : une forme de dévolution}

L'enregistrement de l'œuvre constitue un savoir spécifique et la relation qui va s'établir entre ce savoir et l'élève au cours de l'écoute demeure problématique. Dans le cas de la confrontation à un texte littéraire ou à un document iconographique, il est assez aisé pour un enseignant d'anticiper sur les éléments à percevoir ou à comprendre. Dans le cas de la musique, on ne peut être sûr de ce qui se passe entre l'œuvre musicale et les élèves, puisque la rencontre reste en grande partie subjective, voire « ineffable». Les propos de Jankélévitch qui déclare que chacun peut faire dire aux notes de musique ce qu'il veut (Jankélévitch, 1983) souligne toute la difficulté de construire un savoir collectif à partir de l'écoute de l'enregistrement d'une ouvre musicale. Dans la classe, c'est précisément le produit de la confrontation collective des perceptions individuelles qui constitue le savoir sur lequel se construit la connaissance de l'œuvre. Ainsi, lorsque l'enseignant fait écouter l'œuvre, avec ou sans consigne, il dévolue le jeu aux élèves, puisque l'écoute est un jeu centré sur le savoir. C'est le moment où l'élève doit assumer « une forme de solitude face à l'apprentissage » et doit accepter de "prendre sur soi de se confronter au texte » (Sensevy, 2008, p. 46). Le but de la "réticence didactique ", dont nous en avons montré précédemment un exemple (jeu 1), « est bien de permettre ce rapport de première main » évoqué par Sensevy (2008, p. 46). Toutefois, si lors de l'écoute le jeu est censé être dévolué, nous verrons comment l'enseignant dévoilera à certains moments des éléments de savoir amenant l'élève à « produire des stratégies gagnantes de faible valeur » (Sensevy, 2008, p. 46).

L'œuvre dure 12 minutes. Elle est diffusée 4 fois en entier au cours de la séance, ce qui revient à 48 minutes d'audition dans la séance de 80 minutes. Le choix de ne pas fractionner l'écoute est lié au genre musical à programme. Pour l'enseignant, il est essentiel que l'œuvre soit écoutée dans son ensemble. C'est un moyen de donner du sens à cet objet abstrait en passant par les images évoquées par la musique que seule une écoute globale permet de percevoir.

- « Je pense qu'ils ont senti les différentes séquences, d'où l'intérêt de cette écoute prolongée, c'est certain, bon par contre c'est vrai que ça fait une séquence qui est très longue sur 
une musique mais bon je trouve que ça pouvait être intéressant. » (autoconfrontation.)

- « Oui je trouvais que ça pouvait être intéressant parce que si on séquençait nous-mêmes alors là par contre pour le coup on les guidait, déjà que je trouve que je les guide un petit peu sur il y a plusieurs parties... le but du jeu c'était de leur faire sentir aussi qu'est-ce que ça leur évoquait tout ça parce que je pense que écouter une œuvre, bon y'a l'aspect technique et cetera mais c'est aussi important de se dire qu'a voulu nous dire le compositeur. C'était le but. C'est pour ça qu'on a pris un peu plus de temps là-dessus. » (autoconfrontation.)

La volonté de l'enseignant d'entraîner les élèves à une écoute prolongée est également liée au niveau de la classe (CM2); il déclare qu'il aurait fait autrement avec des plus jeunes élèves. Mais les modalités d'écoute sont surtout relatives au genre musical à programme. En effet, l'enseignant souhaite aborder l'œuvre à travers les intentions de l'auteur. Or, « séquencer » l'écoute revient pour l'enseignant à " guider » les élèves et à faire le travail de description à leur place, conduisant à faire produire des stratégies gagnantes «pauvres en savoir» (Sensevy, 2008). Le PE juge « intéressant », "important » d'amener les élèves à découvrir les intentions de l'auteur à partir de quelques éléments caractéristiques de l'œuvre. Si le contrat initial vise la construction de la représentation générale de l'œuvre, il ne fait aucun doute que pour l'enseignant cette représentation passe par le repérage de ses différentes parties et de son organisation générale (entretien post).

Chacune des quatre écoutes est accompagnée d'une consigne spécifique. Pour la première écoute (jeu 2), la consigne est d'abord implicite. Il s'agit pour les élèves de prendre connaissance de l'œuvre et de dire ensuite ce qu'ils ont entendu. C'est l'écoute « un peu libre » dont parle l'enseignant dans l'entretien post. Bien que cette consigne soit précisée à la fin du jeu 1 par un « indice » (extrait 1 ), le contrat de départ n'est pas véritablement modifié. La définition du jeu qui précède la deuxième écoute (jeu 5) consiste à repérer le nombre de parties et oriente la perception des élèves vers les contrastes qui caractérisent cette œuvre, ce qu'expriment leurs réponses à l'issue de cette deuxième écoute («parce que des fois c'est plus ou moins fort », min 34:32, "ça change », $\min$ 34:50).

La troisième écoute (jeu 7) est une écoute fractionnée et commentée. Elle permet d'identifier et de décrire chacune des parties. La consigne consiste pour les élèves à indiquer à l'enseignant les moments où il doit stopper l'écoute afin de marquer les différentes parties. Mais, au cours de ce jeu, le contrat est modifié puisque les élèves sont peu à peu amenés à répondre à la question de l'enseignant sur les raisons qui l'amènent à stopper lui-même l'enregistrement, alors qu'initialement ce sont eux qui sont censés indiquer et justifier les arrêts de l'enregistrement. L'action de l'enseignant glisse peu à peu de la dévolution à la régulation et la description se fera souvent en simultanéité avec l'écoute de l'enregistrement.

Enfin, la quatrième écoute n'était pas prévue, elle est demandée par un élève et accompagne le travail de synthèse écrite qui consiste à compléter un tableau pour donner un titre à chacune des parties de l'œuvre (jeu 9) : « tu veux l'écouter en même temps? Allez je peux vous le mettre en fond sonore si vous voulez » (min 63:26). Cette quatrième écoute ne modifie pas véritablement le milieu ni le contrat. Elle est proposée en « fond sonore » et on peut penser que même si les élèves entendent l'enregistrement, ils n'y prêtent pas forcément attention et le contrat demeure bien lidentification des dix parties de l'œuvre à partir du texte. En effet, la surcharge d'éléments proposés visuellement (photos et texte) et auditivement (l'enregistrement) peut constituer un obstacle à l'écoute qui apparaît secondaire dans le milieu et relativement au contrat.

\section{Le savoir construit conjointement : les interactions verbales PE / élèves}

L'analyse de l'œuvre s'appuie essentiellement sur trois éléments : le programme, les caractéristiques musicales (thème musical, nuances, tempo, matériau sonore) et l'organisation de l'œuvre. Ces éléments sont en interrelation puisque l'organisation est entièrement dépendante du programme et que c'est la présence, l'absence ou la transformation de certains éléments caractéristiques, notamment le thème musical principal, qui permet de repérer le passage d'une partie à l'autre.

En dehors des informations verbales et non verbales données par le PE, c'est essentiellement à partir de l'identification, par les élèves, des éléments extra-musicaux (thème, tableaux) et musicaux (thème, dynamiques, ruptures, contrastes) que le programme de l'œuvre est mis en évidence au cours des échanges. 


\section{Le programme}

Voyons tout d'abord comment le programme de l'œuvre émerge au cours des échanges. Nous avons remarqué que l'enseignant ne veut pas le dévoiler et en fait un enjeu d'écoute. Pour cela il donne un « indice » et c'est précisément à cet indice que répond l'élève (E2) à l'issue de la première écoute (min 14:55).
C'est à partir de la réponse de E2 que le dialogue s'engage et amène d'autres élèves à faire des propositions. Même si l'enseignant s'inscrit dans une démarche non transmissive et sollicite largement l'expression des élèves, nous remarquons que la mise en évidence des éléments perçus par les élèves est rapidement validée par l'enseignant (effet Jourdain). En effet, le rapprochement avec une ouvre précédemment étudiée (« est-ce que vous pouvez le

Tableau 4. Extrait 3 de la situation, de min 14:43 à min 16:40

\begin{tabular}{|c|c|c|}
\hline \multirow[t]{2}{*}{$14: 43$} & & PE arrête l'écoute, des élèves lèvent la main pour demander la parole \\
\hline & $\mathrm{PE}$ & $\begin{array}{l}\text { Bon alors déjà c'était un p / : le le : cette partie dure à peu près douze minutes c'est bien vous avez } \\
\text { bien écouté ça je vous félicite ça c'est très bien }\end{array}$ \\
\hline \multirow[t]{24}{*}{$14: 55$} & E2 & J'ai trouvé \\
\hline & PE & Qu'est-ce que tu as trouvé? \\
\hline & E2 & Heu : les images qu'on peut mettre dessus \\
\hline & PE & Quelles images tu pourrais mettre dessus? \\
\hline & E 2 & Tu as dit ben / une personne qui se balade dans la forêt \\
\hline & PE & Une personne qui se promène dans la forêt / pourquoi? \\
\hline & E2 & Ben heu \\
\hline & PE & $\begin{array}{l}\text { Non moi j'ai parlé d'un élément naturel alors ça pourrait être une forêt effectivement : mais est-ce } \\
\text { qu'il y a des: }\end{array}$ \\
\hline & E3 & Une cascade \\
\hline & $\mathrm{PE}$ & Une cascade / oui pourquoi une cascade? \\
\hline & E3 & XXXX \\
\hline & E4 & Parce que la musique fait beaucoup de bruit \\
\hline & PE & Ah lorsque la musique fait beaucoup de bruit c'est ça? \\
\hline & E4 & Oui \\
\hline & $\mathrm{PE}$ & Oui \\
\hline & E5 & Après un ruisseau qui coule quand ça fait moins de bruit \\
\hline & $\mathrm{PE}$ & Un ruisseau qui coule \\
\hline & E6 & Des oiseaux \\
\hline & PE & C'est ça \\
\hline & E7 & Un orage qui \\
\hline & Es & $\operatorname{xxxxx}$ \\
\hline & $\mathrm{PE}$ & $\begin{array}{l}\text { Est-ce que vous pouvez le mettre en rapport avec quelque chose que je vous ai fait écouter } \\
\text { une fois lorsque }\end{array}$ \\
\hline & E8 & Oui y’a pas longtemps tu t'en rappelles on avait écouté un ruisseau qui coulait \\
\hline & PE & $\begin{array}{l}\text { Un ruisseau qui coulait vous vous souvenez en relaxation on avait fait l'écoute il y avait } \\
\text { effectivement un ruisseau le cours d'un ruisseau la rivière et cetera et effectivement eh bien voyez } \\
\text { heu : / c'est quand même important / de bien écouter une oeuvre parce que justement cette } \\
\text { œuvre c'est le cours d'une rivière et elle s'appelle La Moldau / La Moldau c'est plus pratique c'est } \\
\text { le nom allemand parce que vous allez voir le nom tchèque c'est la / (prononce tflava) (rire) / c'est } \\
\text { une rivière qui traverse (prend des documents sur son bureau) alors je vais vous montrer là / qui } \\
\text { traverse effectivement / heu : la république Tchèque actuelle (distribue une reproduction de carte à } \\
\text { chaque élève) / voilà / voyez c'est cette rivière et c'est cette rivière que le compositeur a voulu } \\
\text { décrire / d'accord / alors qu'est-ce que vous avez remarqué sur ce morceau sur ce poème? }\end{array}$ \\
\hline
\end{tabular}


mettre en rapport avec quelque chose que je vous ai fait écouter...», min 15:40), tout comme la carte de géographie distribuée et les propos qui l'accompagnent (« voyez c'est cette rivière que le compositeur a voulu décrire », min 16:35) mettent en évidence la volonté de l'enseignant de reconnaître, dans la réponse des élèves, la «stratégie gagnante » (Sensevy, 2007, p. 21).

\section{L'organisation chronologique}

La situation d'écoute observée montre que l'essentiel des tâches d'analyse consiste pour les élèves à mettre en lien le programme avec l'organisation de l'œuvre. La Moldau a été choisie pour cette raison : « c'est une des plus faciles d'accès, [...], on peut dégager facilement les dix parties, elle leur parle en général » (entretien post). Cet enjeu arrive très vite dans la séance et c'est celui des trois écoutes qui vont se succéder après un premier recueil des impressions à la suite de la première écoute.

Différentes interactions confirment que la question de l'organisation en lien avec le programme et les éléments musicaux remarquables (thème, nuances, instruments) est l'objectif principal de cette écoute. Dans l'extrait 4, on peut voir l'action de l'enseignant pour atteindre son objectif. Si l'attitude générale de l'enseignant est de ne pas dévoiler l'œuvre et ses caractéristiques (réticence), il oriente très vite les échanges sur la mise en évidence des différentes parties de l'œuvre, à tel point que l'on peut voir dans la manière de définir la deuxième écoute (jeu 4) en glissant des réponses des élèves (min 16:50) à la

Tableau 5. Extrait 4 de la situation, de min 16:50 à min 17:22 et de min 20:46 à min 21:50

\begin{tabular}{|c|c|c|}
\hline $16: 50$ & PE & $\begin{array}{l}\text { (poursuit la distribution du document) Ah : oui c'est avec des instruments de musique bien sûr c'est } \\
\text { un poème symphonique / d'accord c'est même tout un orchestre / mais qu'est-ce que vous avez } \\
\text { remarqué est-ce que / il est TOUT LE TEMPS sur la même tonalité ? }\end{array}$ \\
\hline & Es & Non \\
\hline & $\mathrm{PE}$ & Sur le même rythme \\
\hline & Es & Non \\
\hline & $\mathrm{PE}$ & Non / \\
\hline & E & il change tout le temps il a pas de: \\
\hline & PE & Oui : Louise qu'est-ce que tu dis pardon \\
\hline & E & Il change tout le temps \\
\hline & $\mathrm{PE}$ & $\begin{array}{l}\text { Il CHANGE TOUT LE TEMPS je ne sais pas si il change tout le temps mais on peut peut- } \\
\text { être effectivement dégager plusieurs? }\end{array}$ \\
\hline & Es & sons \\
\hline & E & Quoi / si ça change / plusieurs \\
\hline & Es & Sons \\
\hline $17: 22$ & $\mathrm{PE}$ & $\begin{array}{l}\text { Plusieurs / ben plusieurs parties tout simplement bien sûr plusieurs parties / alors on va le } \\
\text { réécouter / encore } 11 \text { minutes hein / vous allez réécouter mais cette fois-ci / en ayant en tête qu'il } \\
\text { s'agit effectivement d'un cours d'eau / d'accord / ... }\end{array}$ \\
\hline \multirow[t]{2}{*}{$20: 46$} & $\mathrm{PE}$ & $\begin{array}{l}\text {...alors j'avais prévu encore deux écoutes / et une troisième avec des pauses mais peut-être : } \\
\text { puisque vous avez bien trouvé par vous-mêmes des choses / on va peut-être travailler direc- } \\
\text { tement en binôme / par deux hein / par deux ou trois / et vous allez essayer / de compter / les } \\
\text { différentes parties de l'œuvre / alors pour vous aider / je vous donne un petit indice / vous n'avez } \\
\text { pas besoin de papier vous n'avez pas besoin de crayon / les doigts de la main suffisent / donc à } \\
\text { chaque fois que pour vous il y a une partie différente (fait des gestes qui marquent la séparation) / } \\
\text { vous la comptez (fait des gestes exprimant une action mentale) après on en discute collectivement / } \\
\text { d'accord / c'est bon / on réécoute? }\end{array}$ \\
\hline & E & Oui \\
\hline $21: 50$ & & PE Démarre l'écoute \\
\hline
\end{tabular}

Les passages en majuscules indiquent une accentuation de la voix. 
question des différentes parties de l'œuvre, un effet «Topaze» (Sensevy, 2007, p. 21). Par exemple, lorsqu'il s'appuie sur la réponse de Louise pour introduire sa nouvelle consigne d'écoute («Il CHANGE TOUT LE TEMPS je ne sais pas si il change tout le temps mais on peut peut-être effectivement dégager plusieurs ? »), l'enseignant oriente l'attention des élèves sur les informations qu'il attend.

\section{Les éléments musicaux repérés : thème musical, nuances, matériau sonore}

Voyons maintenant comment l'identification des éléments musicaux caractéristiques devient le moyen de dégager les différentes parties de l'œuvre. Par exemple, le thème principal de La Moldau est remarqué par un élève ( « il y a un air de chanson mais c'est toujours le même », min 34:57). L'enseignant veillera à ce que tous les élèves identifient le thème principal en le fredonnant.

De la même manière que dans l'exemple précédent, la mise en évidence du thème permet à l'enseignant de relancer la question centrale concernant l'identification des différentes parties (effet Topaze) : « oui on est toujours sur le même thème / donc on peut identifier les parties parce que / les mélodies ne sont pas les? $\gg(\min 44: 23$.)

D'autres éléments musicaux sont abordés de la même façon et c'est notamment à partir de la question concernant le nombre de parties de l'œuvre et la manière de les identifier que ces données émergeront des réponses des élèves. Seront pointées à plusieurs reprises, les nuances, qui contribuent largement à souligner les ambiances ou les changements de tableaux, et la densité de certains éléments du programme, comme le montre l'échange entre plusieurs élèves dans l'extrait 3 : « une cascade »

Tableau 6. Extrait 5 de la situation, de min 34:15 à min 36:36

\begin{tabular}{|c|c|c|}
\hline \multirow[t]{9}{*}{$34: 15$} & $\mathrm{PE}$ & $\begin{array}{l}\text { Est-ce que vous : chut : oui / alors c'est vrai que c'est une ouvre qui pourrait être romantique } \\
\text { parce que effectivement les sentiments sont exaltés (fait un geste de grandeur) : notamment à } \\
\text { certains passages / bon alors on va reprendre dans l'ordre / Qu'est-ce qui vous permet d'identifier } \\
\text { différentes parties? }\end{array}$ \\
\hline & E & Parce que des fois c'est plus ou moins fort \\
\hline & $\mathrm{PE}$ & Alors il y a le volume /oui donc peut-être le nombre d'instruments / le choix des instruments \\
\hline & E & Peut-être aigu ou grave / aigu ou grave \\
\hline & $\mathrm{PE}$ & $\begin{array}{l}\text { Non mais il y a d'autres choses quand même qui permettent (interroge un élève qui lève la main) / } \\
\text { oui }\end{array}$ \\
\hline & $\mathrm{E}$ & Ça change \\
\hline & $\mathrm{PE}$ & Ça change \\
\hline & E & Des nuances \\
\hline & $\mathrm{PE}$ & Oui ? \\
\hline \multirow[t]{6}{*}{$34: 57$} & E & $\begin{array}{l}\text { Aussi il y a un air de chanson mais c'est toujours le même mais des fois il baisse il aug- } \\
\text { mente }\end{array}$ \\
\hline & $\mathrm{PE}$ & $\begin{array}{l}\text { Ah / il y a un : comment on appelle ça? (fait le geste de retour avec la main) alors un air de } \\
\text { chanson : non }\end{array}$ \\
\hline & Es & $\begin{array}{l}\text { XXXX (réponses inaudibles desquelles émergent le terme " thème " que le PE n'entend pas) (auto- } \\
\text { confrontation) }\end{array}$ \\
\hline & $\mathrm{PE}$ & $\begin{array}{l}\text { Non / non quelque chose qui revient toujours / effectivement est-ce que tu peux nous la chanter } \\
\text { (s'adresse à un élève) / cette chanson / qui revient tout le temps }\end{array}$ \\
\hline & E & Fredonne un air peu ressemblant \\
\hline & $\mathrm{PE}$ & $\begin{array}{l}\text { Non c'est pas ça / non / bon je vais vous le chanter (fredonne le thème en battant la mesure avec la } \\
\text { main) / c'est un / comment on appelle ça / on appelle ça le thème / on va dire c'est un thème qui } \\
\text { revient / d'accord / d'ailleurs ce thème Smetana mais on le verra après / il l'a repris d'une chanson } \\
\text { populaire / une chanson populaire bohémienne / et ce thème il a été repris aussi on le verra dans } \\
\text { une prochaine séance / sur le thème pour l'hymne national d'Israël / on verra pourquoi oui / mais } \\
\text { ça dans une prochaine séance / donc combien vous avez trouvé de parties? }\end{array}$ \\
\hline
\end{tabular}


(min 15:11), « parce que la musique fait beaucoup de bruit » (min 15:21), « après un ruisseau qui coule quand ça fait moins de bruit » (min 15:27).

La présence de certains instruments est également considérée d'un point de vue descriptif, en lien avec le programme de l'œuvre, par exemple la flûte du début de l'œuvre ( « une flûte / qu'est-ce qu'elle vous évoque cette flûte? », min 39:20), ou encore le cor. ment des contrastes d'intensité, d'orchestration, de tempo qui caractérisent cette œuvre, l'idée d'une organisation en plusieurs parties pouvait émerger, même si l'analyse n'aurait sans doute pas permis d'aboutir à une description complète et précise en si peu de temps, comme l'envisage ici l'enseignant.

Tableau 7. Extrait 6 de la situation, de min 45:00 à min 45:46

\begin{tabular}{|l|l|l|}
\hline $45: 00$ & PE & (arrête la musique) Louise me dit oui de stopper / qu'est-ce qu'on entend? \\
\hline & Es & La trompette / le violon XXX \\
\hline & PE & non c'est pas exactement une trompette \\
\hline & Es & (Réponse collective au cours de laquelle on devine le mot cor) \\
\hline & PE & Pardon \\
\hline & E & Un cor un truc comme ça \\
\hline & PE & Alors : des trucs comme ça je sais pas \\
\hline & E & C'est un cor \\
\hline & PE & $\begin{array}{l}\text { Un cor / un cor / je dis d'accord pour le cor / heu : le cor en général / c'est un instrument qui } \\
\text { est comment ? }\end{array}$ \\
\hline & Es & Grave et gros XXXX \\
\hline & PE & Oui / en rond / oui oui / on l'utilise pour quoi ? souvent \\
\hline & E & La chasse \\
\hline & PE & La chasse / la chasse à courre \\
\hline & E & Ah oui le cor de chasse \\
\hline & PE & Oui alors ça pourrait peut-être? \\
\hline & PE & Alors on y va (redémarre la musique) \\
\hline
\end{tabular}

Ces deux derniers exemples (extraits 5 et 6) montrent encore, dans la manière de valider les réponses (effet Jourdain) et d'introduire les questions (effet Topaze), comment l'enseignant amène les élèves à produire les réponses attendues, alors qu'il dit au départ ne pas vouloir guider l'écoute. Néanmoins, même si l'enseignant déclare cette œuvre " accessible » (entretien post), l'identification de ces différentes parties nous apparaît complexe sur le plan didactique et musical. D'ailleurs, lorsque les élèves doivent identifier le nombre de parties à l'issue de la deuxième écoute, les réponses varient très largement. À la question « combien avez-vous trouvé de parties ? ( $\min 36: 36)$, les élèves déclarent dans une sorte de surenchère collective : "vingt ", « six ", « quinze », « vingt-cinq ». À tel point que lorsque le nombre « dix » est avancé, l'enseignant répond «Dix / c'est au hasard que tu dis ça » (min 36:58). En partant des éléments perçus par les élèves et notam-
LE PROGRAMME : UN ÉLÉMENT CENTRAL POUR ACCÉDER À LA CONNAISSANCE DE L'GUVRE

À l'observation de la situation d'enseignementapprentissage de l'écoute de La Moldau de Smetana, nous avons pointé l'importance du programme en tant que savoir sur l'œuvre dans l'objectif de l'enseignant qui exclut une approche uniquement fondée sur le ressenti des élèves. C'est l'analyse des caractéristiques de l'action didactique en termes de mésogenèse, topogenèse et chronogenèse qui nous permet de mieux comprendre l'action de l'enseignant.

\section{"Mésogenèse " : la co-construction par les différents acteurs du milieu d'apprentissage}

Les écoutes de l'enregistrement de l'œuvre et les échanges verbaux qui en découlent contribuent 
fortement à la création du milieu didactique dans une séance consacrée à la découverte et à la connaissance d'une ouvre musicale. C'est une des caractéristiques des milieux dans lesquels l'élève fait « l'expérience de l'œuvre en réception ", où le savoir se construit à partir des deux tâches : écouter et dire. C'est « la parole sur l'œuvre » (Chabanne \& Dufays, 2011) qui nous permet d'en mesurer l'avancée.

L'analyse des interactions montrent que les élèves sont rompus à l'exercice de l'écoute. Ils sont capables d'écouter plusieurs fois une œuvre longue et s'expriment presque spontanément à l'issue des écoutes sans que soit relancée la question, comme on peut l'observer au début du jeu 3, ("j'ai trouvé », min 14:55). Les interactions mettent également en évidence les références musicales des élèves concernant un certain nombre de notions, comme le thème musical, les nuances, le matériau sonore. C'est un vocabulaire et des notions qu'ils semblent avoir l'habitude de manier. Les élèves sont intéressés par l'activité ; ils n'hésitent pas à poser des questions pertinentes ("ça veut dire quoi symphonique? », min 0:57). L'analyse des différents extraits montre encore que c'est la réponse des élèves aux questions de l'enseignant qui permet de faire émerger les éléments caractéristiques de l'œuvre.

L'enseignant qui vise la construction des savoirs sur l'œuvre par les élèves donne peu d'indices au départ (min 0:35). Mais très vite il va apporter des éléments supplémentaires sur le contexte historique et géographique de l'œuvre (min 1:13) qui dépassent très largement la réponse qu'il souhaite apporter à la question de l'élève sur le sens du mot symphonique. Il juge ces éléments « nécessaires pour aider les élèves à identifier les éléments importants de l'œuvre » (autoconfrontation), pourtant, il ne les donnait pas au départ. On constate également, au vu de l'indice donné au début (« il parle d'un élément / de son pays / et cet élément c'est un élément naturel $», \min 2: 18$ ) et des outils didactiques distribués au cours de la séance (cartes, texte, tableau) que les informations importantes à transmettre aux élèves sont relatives au programme de l'œuvre (« en tous cas dans le contexte de cette œuvre-là puisque c'est un poème symphonique », autoconfrontation). Nous observons que les différents supports qui vont modifier le milieu didactique interviennent le plus souvent après l'émergence du savoir et ont une fonction essentiellement démonstrative en vue de définir, de réguler le jeu ou d'institutionnaliser le savoir. On a vu aussi que toutes les informations transmises par l'enseignant avaient pour but de relier l'œuvre à des éléments extra-musicaux, le plus souvent, l'existence de la rivière évoquée (la carte de géographie), le programme (le texte sur lequel le compositeur s'est appuyé), les différents tableaux de l'œuvre (les photos projetées sur le TNI). Dans les trois cas, le PE insiste sur le caractère " actuel », « authentique », « existant» des objets de savoir apportés et qui permettent de donner un aspect matériel à l'œuvre musicale en lien avec les intentions de l'auteur :

« c'était bien qu'ils l'aient cette feuille pour qu'ils voient que cette œuvre qui est une œuvre artistique quelque part elle a quelque chose d'intemporel parce que : la rivière est encore là et que : c'était une carte : actuelle que je leur avais donnée donc : voilà c'est une œuvre qui fait à la fois partie du patrimoine et : la rivière est encore là / ça je voulais faire ce lien-là » (autoconfrontation).

Pour l'enseignant, la relation avec la réalité et l'actualité historiques et géographiques constitue un élément important dans la transmission du patrimoine, tout comme le lien avec le programme et l'organisation de l'œuvre. Autant d'éléments concrets qui laissent peu de place au ressenti émotionnel de l'élève que l'enseignant déclare pourtant être un aspect habituellement mis en avant (autoconfrontation), et aux éléments strictement musicaux comme les nuances, le thème et l'orchestration. Par exemple, lorsque l'élève Louise déclare qu'« il change tout le temps» (min 17:02), l'enseignant aurait pu s'appuyer sur les changements évoqués pour aborder ces éléments musicaux de l'œuvre (nuances, orchestration, tempo, densité) vers lesquels les élèves semblent vouloir aller. Mais c'est encore pour orienter l'analyse sur l'organisation de l'œuvre que le PE reprend la déclaration de Louise : "Il CHANGE TOUT LE TEMPS /... / on peut peut-être effectivement dégager plusieurs [parties] ?» (min 17:07).

\section{La « topogenèse » : la responsabilité dans l'avancée du savoir}

Les extraits analysés illustrent bien les places respectives de l'enseignant et des élèves. Par exemple, dans l'extrait 1 , l'enseignant souhaite visiblement ne pas orienter l'écoute de ses élèves et les laisser 
découvrir par eux-mêmes les intentions de l'auteur et les caractéristiques musicales de l'œuvre. Il juge d'ailleurs que les informations qu'il donne à la suite de la question de El sont un peu longues : « je dirai que j'ai été long en revoyant la vidéo » (autoconfrontation). Les informations données sur l'œuvre se concluent par un "petit indice » illustrant bien que c'est l'élève qui est mis en posture de recherche. C'est ce que montrent les premières prises de paroles des élèves qui répondent très logiquement à l'« indice » : « j'ai trouvé... les images qu'on peut mettre dessus » (min 14:55). L'enseignant procède de la même façon pour faire émerger la présence du thème musical (extrait 4) et de différents éléments caractéristiques de l'œuvre (extraits 5 et 6 ), toujours en lien avec le programme. Il souhaite avoir un rôle de guide pour aider les élèves à construire le savoir : « les aider à identifier les éléments qui me semblaient importants dans cette œuvre » (autoconfrontation). En ce qui concerne les écoutes, elles sont de la responsabilité de l'enseignant qui en détermine la durée (ici, la totalité), l'objectif (identifier les différents tableaux et donc l'organisation de l'œuvre) et le nombre («alors j’avais prévu encore deux écoutes / et une troisième avec des pauses mais peut-être : puisque vous avez bien trouvé par vous-mêmes... », min 20:44) qu'il décide de réduire au vu de l'avancée du savoir. Seule la quatrième écoute, initialement prévue puis supprimée par l'enseignant sera réclamée par un élève, même si elle n'est pas exploitée et se limite à une diffusion « en fond sonore» (min 63:21).

Ainsi, l'analyse didactique nous permet de distinguer deux postures topogéniques : $1^{\circ}$ celle de l'enseignant qui cherche à faire émerger le savoir en faisant écouter l'œuvre en entier plusieurs fois et produire aux élèves les réponses aux questions qu'il pose et, $2^{\circ}$ celle des élèves qui écoutent l'enregistrement de l'œuvre pour identifier, repérer les éléments caractéristiques et les exposer oralement en réponse aux questions de l'enseignant. Toutefois, l'analyse montre à plusieurs reprises un enseignant qui reprend rapidement la responsabilité dans l'avancée du savoir, notamment lorsqu'il s'appuie sur les propositions des élèves ou les documents qu'il introduit pour définir ou réguler le jeu. C'est par exemple le cas, à l'issue du jeu 3, quand il initie la consigne de la deuxième écoute, alors qu'un élève vient de faire remarquer que c'est une œuvre instrumentale : " oui c'est avec des instruments de musique bien sûr / c'est un poème symphonique / d'accord c'est même tout un orchestre / qu'est-ce que vous avez remarqué est-ce que / il est TOUT LE TEMPS sur la même tonalité ? » (min 16:50). Mais c'est encore plus visible lors de la troisième écoute (jeu 7) où les élèves sont censés indiquer les changements de parties. L'indication des arrêts de l'enregistrement appartient au départ au topos des élèves ( $«$ vous me dites quand je pose hein et pourquoi » (min 37:58), mais dès l'approche de la troisième partie de l'œuvre, le PE, par un geste qu'il accompagne d'une question, indique le changement : « et qu'est-ce qui se passe là ? » (min 42:30). L'analyse de la chronognenèse qui suit montre le lien évident entre la gestion du temps et la gestion des topos.

\section{«Chronogenèse " : la gestion des temporalités d'apprentissage}

En utilisant le néologisme " chronomaître », Sensevy (2001) insiste sur une des fonctions primordiales de l'enseignant que représente la gestion du temps didactique. C'est précisément la gestion de ce temps qui amène l'enseignant à accélérer à certains moments la chronogenèse, au point de modifier le topos des élèves, comme on vient de le voir.

L'analyse chronogénétique montre bien une analyse de l'œuvre étudiée dans cette classe de CM2 centrée sur l'élément extra-musical. La séance est articulée autour des quatre écoutes de l'enregistrement avec une évolution du questionnement et des éléments de savoir qui vont du thème de l'œuvre ( " un élément naturel ») aux dix parties de l'œuvre correspondant au poème présenté par l'enseignant.

L'enseignant déclare qu'il est important d'écouter plusieurs fois l'œuvre étudiée et que ce sont ces écoutes successives qui permettent une évolution dans l'avancée du savoir : " bon d'abord on écoute un peu de façon libre et puis petit à petit on essaie de recentrer un petit peu les choses » (entretien post). Dans l'entretien d'autoconfrontation, il remarque que les élèves sont partis très vite «sur un scénario » contrairement à leurs habitudes où ils s'expriment d'abord sur leur ressenti. Si l'enseignant remarque cette orientation prise par les élèves, l'observation montre qu'il en est responsable. En effet, l'analyse de la situation met en évidence des accélérations de la chronogenèse assez fréquentes. C'est d'abord, comme nous venons de le voir, au cours du jeu 1 lorsque l'enseignant oriente la première écoute sur 
le thème extra-musical dès la consigne de départ. Ensuite, quand les élèves décrivent ce qu'ils ont entendu au cours du jeu 3, l'enseignant fait le lien avec une œuvre précédemment entendue avant d'enchaîner rapidement sur une deuxième consigne d'écoute: « est-ce qu'il est tout le temps sur la même tonalité ?» (min 16:50), limitant la durée du jeu 3, pourtant fondamental dans la démarche, à une minute (de min 14:43 à min 15:43). Au cours du jeu qui suit, le PE précise qu'il passe directement à la troisième écoute, en raison des réponses positives des élèves : "puisque vous avez bien trouvé par vous-mêmes des choses / on va peut-être travailler directement en binôme » (min 20:46). Cette seconde écoute effective, mais troisième dans la programmation de l'enseignant, consiste pour les élèves à se mettre en binôme pour compter le nombre de parties. L'analyse montre encore une accélération de la chronogenèse au cours de la troisième écoute qui consiste initialement à fractionner l'enregistrement en vue de montrer les différentes parties de l'œuvre (jeu 7). Mais, dès la cinquième partie, l'analyse se fait simultanément avec l'écoute de l'enregistrement que l'enseignant ne prend plus le temps de stopper pour poser les questions ou apporter des commentaires (tableau 1).

L'objectif de la séance vise la description de l'œuvre à travers le programme qui est à l'origine de sa composition. L'analyse montre, comment d'une démarche maieutique (Mili, 2011) envisagée au départ, l'enseignant qui déclare pourtant ne pas vouloir «trop guider les élèves » (autoconfrontation) adopte peu à peu un topos en « surplomb», en modifiant le milieu par l'introduction d'informations verbales et non verbales, non toujours prévues, et la forme des questions qui devient de plus en plus fermée, concomitantes à des accélérations chronogénétiques fréquentes.

Si le contrat de départ vise la construction du savoir par l'élève à partir de ce qu'il perçoit et identifie au cours des différentes écoutes, l'analyse montre la responsabilité de l'enseignant dans l'émergence d'un certain nombre de savoirs, notamment ceux liés au programme de l'œuvre. Toutes les consignes d'écoute visent à faire converger la représentation des élèves vers les éléments relatifs au programme, et c'est pour valider et institutionnaliser ces mêmes savoirs que l'enseignant introduit un certain nombre d'outils didactiques écrits et iconographiques, n'hésitant pas ainsi à modifier le milieu.
La théorie de l'action conjointe en didactique (TACD) nous apparaît particulièrement propice à l'analyse de l'action didactique en écoute musicale qui consiste à faire émerger et construire le savoir sur l'œuvre à partir de ce que les élèves perçoivent et ressentent à son contact. Il s'agit pour l'enseignant de créer le milieu favorable à l'écoute puis à l'expression et la confrontation des différentes représentations que les élèves ont pu se faire de l'œuvre. Il s'agit bien d'une action didactique nécessairement conjointe, dont les jeux, spécifiés à un enjeu de savoir, se succèdent au fil d'une relation didactique (Sensevy, 2007). Mais, si cette théorie nous a permis d'inférer certains déterminants, dont elle postule l'existence du côté de l'enseignant, elle ne permet pas toujours de comprendre les pratiques du côté des élèves. Pour accéder à une intelligibilité plus complète de l'action didactique qui conduit à faire co-construire le savoir sur une ouvre au cours d'une séance d'écoute, il serait nécessaire d'accéder à certains déterminants de l'action didactique liés aux élèves, comme par exemple, tout ce qui se passe entre l'œuvre et les élèves, ou encore l'écart entre ce que ces derniers perçoivent et ce qu'ils disent.

\section{CONCLUSION}

L'écoute musicale à l'école vise la connaissance d'un certain nombre d'œuvres. Cette connaissance s'élabore à partir des écoutes répétées au cours desquelles l'élève se construit une représentation personnelle de l'œuvre, qu'il partage ensuite et confronte avec celle de ses pairs. C'est la confrontation des représentations que chacun a pu se forger en écoutant l'œuvre qui permet de construire « une réponse assumée par plusieurs élèves » (Grandaty, 2005), si ce n'est par tous, plutôt qu'une description et une analyse de l'œuvre imposée de l'extérieur. Mais quelles connaissances des œuvres est censée transmettre l'école? Comment une ouvre instrumentale prend-t-elle la forme d'un objet d'apprentissage ? Comment émerge-t-elle dans la classe en tant qu'œuvre? Quelles représentations en construisent conjointement l'enseignant et les élèves ? En analysant une séance relative à l'écoute musicale d'une œuvre appartenant au répertoire assez couramment mis en ouvre par les enseignants de l'école primaire (Maizières, 2014), nous avons voulu répondre à un certain nombre de ces questions. 
L'analyse didactique de la séance observée dans cette étude met en évidence une situation d'écoute qui se caractérise par l'articulation des écoutes de l'enregistrement de l'œuvre avec les interactions verbales entre l'enseignant et les élèves. Ainsi, l'analyse montre:

- du côté de la mésogenèse, l'importance de chacun des éléments du milieu didactique, à savoir, l'œuvre (ses caractéristiques, sa durée, sa présence sonore dans la classe), les élèves (leurs compétences à écouter et à réagir, les savoirs exprimés à propos de l'œuvre, leurs références musicales) et l'enseignant, notamment les savoirs qu'il apporte suite à la question d'un élève ou encore ceux qu'il transmet par l'intermédiaire des documents donnés aux élèves à l'issue de leurs réponses ;

- du côté de la topogenèse, le rôle de chacun des acteurs dans l'avancée du savoir, soit l'enseignant qui définit, informe, dévolue, guide, démontre, valide, et les élèves qui écoutent, expriment leur perception de l'œuvre, décrivent les éléments perçus, mais surtout répondent aux indices et aux questions que pose l'enseignant ;

- du côté de la chronogenèse, comment la représentation de l'œuvre évolue, de sa découverte sonore à la mise en évidence collective du programme complet de l'œuvre et de son organisation, dans un cadre temporel accéléré par l'enseignant à plusieurs reprises.

L'analyse nous permet donc de répondre à la question des savoirs qui sont censés participer à la connaissance de l'œuvre étudiée. Il s'agit du programme, de son organisation et des éléments caractéristiques relatifs au thème musical et aux paramètres du son (durée, hauteur, intensité et timbres), fondant principalement l'analyse sur les images évoquées par la musique à travers les contrastes de nuances, d'orchestration (objectif déclaré pour la séance suivante) et de tempo. L'analyse nous permet également de décrire et de comprendre la place de chacun des acteurs dans l'émergence et la co-construction des savoirs liés à cette œuvre. Certes, cette étude s'appuie sur un cas et les résultats ne peuvent être généralisés, toutefois l'analyse didactique montre comment d'une démarche maieutique envisagée au départ, l'enseignant adopte peu à peu une position en « surplomb» dans le but de faire émerger le savoir sur l'œuvre à partir de l'élément extra-musical, alors que ce n'est pas forcément ce que les élèves perçoivent et ressentent spontanément à l'écoute.

D'autres études s'intéressent aux questions de transmission-réception des œuvres d'art en milieu scolaire (Chabanne \& Dufays, 2011), plus particulièrement dans le domaine de la musique (Mili \& Rickenmann, 2004 ; Mili, 2011 ; Vendramini, 2012). C'est la forme " abstraite», " intangible », « immatérielle », « ineffable » du savoir de référence dans ce cas qui est montrée comme un facteur de difficulté majeur dans la mise en place de cet enseignement. Autant de mots pour désigner les caractéristiques d'un objet présent dans la classe sous la forme d'une trace sonore s'appuyant sur un langage non signifiant. De plus cet objet appartient au domaine du loisir dans la société et c'est un enseignant, non spécialiste le plus souvent, qui est censé lui donner la forme d'un savoir scolaire.

L'analyse de la séance concernant l'écoute du poème symphonique La Moldau de Smetana montre que c'est le programme qui devient l'élément " molaire " de son étude dans la classe de CM2 observée. C'est précisément cet élément extramusical qui lui donne le caractère « accessible » dont parle l'enseignant. C'est donc autour du programme et de ses dérivés (l'organisation de l'œuvre et certains aspects musicaux qui la soulignent comme le thème, les nuances, etc.) que s'organise la séance qui fait alterner les écoutes de l'œuvre et le discours sur cette écoute.

Une étude antérieure montre que les cuvres à programme ou descriptives sont les plus écoutées dans les classes de primaire (Maizières, 2014). Les résultats de la présente étude nous permettent de faire l'hypothèse que c'est l'élément extra-musical qui, dans le cas d'une musique à programme représente le moyen d'accéder à la compréhension et au sens de l'œuvre pour un enseignant non spécialiste, et moins les éléments musicaux caractéristiques. Qu'en est-il alors de l'étude des œuvres instrumentales non descriptives à l'école primaire? Peut-on faire l'hypothèse que dans ce cas, ce sont davantage les sentiments du récepteur qui sont pris en compte et que c'est à partir des émotions positives ou négatives vécues au cours de l'écoute et confrontées à l'issue de celle-ci que la connaissance de l'œuvre se co-construit dans la classe? 


\section{NOTES}

1. Vltava (La Moldau) fait partie d'un poème symphonique en six mouvements : Má Vlast (Ma Patrie) (https:// fr.wikipedia.org/wiki/Ma Patrie), datant de 1879. Dans la suite du texte, nous donnerons le titre dans sa traduction allemande courante : La Moldau.

\section{RÉFÉRENCES}

Amade-Escot, C., \& Venturini, P. (2009). Le milieu didactique : d'une étude empirique en contexte difficile à une réflexion sur le concept. Éducation \& didactique, 3(1), 7-43.

Amade-Escot, C. (2014). De la nécessité d'une observation didactique pour accéder à l'épistémologie pratique des professeurs. Recherches en Éducation, (19), 18-29.

Bain, D. (2002). Quelques réflexions sur l'utilité de la théorie pour la pratique. Dans M. Wirthner \& M. Zulauf (dir.), À la recherche du développement musical (p. 325340). Paris, France : L'Harmattan.

Brousseau, G. (1998). Théorie des situations didactiques. Grenoble, France : La Pensée Sauvage.

Chabanne, J.-C., \& Dufays, J.-L. (2011). Parler et écrire sur les œuvres littéraires et artistiques : contours et enjeux d'une problématique. Repères, (43). Repéré à http://reperes.revues.org/207

Chabanne, J.-C., Parayre, M., Villagordo, E., \& Dequin, P. (2011). Premiers pas dans la parole sur l'œuvre : observer, interpréter et guider les conduites langagières comme compétence professionnelle. Repères, (43). Repéré à http://reperes.revues.org/218

Forest, D., \& Baltézat-Batellier, P. (2013). Apprentissage d'une pratique instrumentale en orchestre à l'école : une approche didactique. Éducation et Didactique, 7(3), 79-96.

Grandaty, M. (2005). De l'influence des tâches langagières sur les difficultés d'apprentissage. Dans L. Talbot (dir.), Pratiques d'enseignement et difficultés d'apprentissage (p. 49-60). Paris, France : Éditions Erès.

Jankélévitch, V. (1983). La musique et l'ineffable. Paris, France : Éditions du Seuil.

Leblanc, S., Ria, L., \& Veyrunes, P. (2013). Analyse vidéo de situations d'enseignement dans le programme du cours d'action. Dans ViSA : Instrumentation de la recherche en éducation. (2013). Paris, France : Éditions de la Maison des sciences de l'homme. Repéré à http://www. books.openedition.org/editionsmsh/1959, ISBN 9782735116218.

Maizières, F. (2012). Éducation musicale à l'école primaire : quels savoirs enseignés ? Éduquer / Former, 43(1), 1334.
Maizières, F. (2014). Lécoute musicale à l'école primaire : les œuvres que les enseignants déclarent enseigner. Revue des sciences de l'éducation, 40(3), 537-556.

Mialaret, J.- P. (2001). Vers une didactique de l'écoute musicale à l'école. Jalons introductifs. Dans M. Imberty (dir.), De l'écoute à l'œuvre (p. 99-112). Actes du colloque tenu en Sorbonne les 19 et 20 février 1999. Paris, France : L'Harmattan.

Mili, I. (2007). Recevoir une œuvre donnée en création : analyse d'une démarche didactique. Recherche en Éducation Musicale, (25), 223-236.

Mili, I. (2011). Une maieutique de l'écoute musicale en classe. Repères, (43). Repéré à http://reperes.revues. org/227

Mili, I., \& Rickenmann, R. (2004). La construction des objets culturels dans l'enseignement artistique et musical. Dans C. Moro \& R. Rickenmann, Situation éducative et significations (p. 165-196). Bruxelles, Belgique : De Boeck Supérieur.

Ministère de l'Éducation nationale (MEN, 2007). Qu'est-ce que l'écoute? Repéré à http://media.eduscol.education. fr/file/ecole/27/0/ecoute 116270.pdf

Ministère de l'Éducation nationale (MEN, 2008a). Les nouveaux programmes de l'école primaire. Horaires et programmes d'enseignement de l'école primaire, Bulletin officiel, hors-série $\mathrm{n}^{\circ} 3$ du 19 juin 2008.

Ministère de l'Éducation nationale (MEN, 2008b). Histoire des arts, école primaire. Listes d'exemples d'œuvres. Repéré à http://eduscol.education.fr/histoiredesarts

Ministère de l'Éducation nationale de l'Enseignement supérieur et de la Recherche (MENESR, 2006). Décret $\mathrm{n}^{\circ} 2006-830 \mathrm{du} 11$ juillet 2006 relatif au socle commun de connaissances et de compétences et modifiant le code de l'éducation. Journal Officiel du 12 juillet 2006.

Schubauer-Leoni, M.-L. (2008). La co-construction des savoirs. Dans N. Wallian, M.-P. Poggi \& M. Musard (dir.), Co-construire des savoirs : les métiers de l'intervention dans les APSA (p. 67-86) Besançon, France : Presses universitaires de Franche-Comté.

Sensevy, G. (2001). Théorie de l'action et action du professeur. Dans J.-M. Baudouin et J. Friedrich (dir.), Théories de l'action et éducation (p. 203-224). Bruxelles, Belgique : De Boeck supérieur.

Sensevy, G. (2007). Des catégories pour décrire et comprendre l'action didactique. Dans G. Sensevy \& A. Mercier (dir.), Agir ensemble. L'action didactique conjointe du professeur et des élèves (p. 13-49). Rennes, France: Presses universitaires de Rennes.

Sensevy, G., \& Mercier, A. (2007). Agir ensemble : l'action didactique conjointe. Dans G. Sensevy \& A. Mercier (dir.), Agir ensemble. Laction didactique conjointe du professeur et des élèves (p. 187-211). Rennes, France : Presses universitaires de Rennes.

Sensevy, G. (2008). Le travail du professeur pour la théorie de l'action conjointe en didactique, Recherche et formation, (57), 39-50.

Sensevy, G. (2011). Le sens du savoir. Éléments pour une théorie de l'action conjointe en didactique. Bruxelles, Belgique : De Boeck. 
Terrien, P. (2006). L'écoute musicale au collège, fondements anthropologiques et psychologiques. Paris, France : L'Harmattan.

Vendramini, C. (2012). Lécoute musicale dirigée à l'école : pour enseigner quelle parole sur l'œuvre d'art ? Colloque international "Formes d'éducation et processus d'émancipation", (p. 6-15). Université de Bretagne Occidentale. Repéré à http://esup.espe-bretagne.fr/colloque cread 2012/paper submission/Vendramini.pdf

Venturini, P., \& Tiberghien, A. (2012). La démarche d'investigation dans le cadre des nouveaux programmes de sciences physiques et chimiques : étude de cas au collège. Revue française de pédagogie, (180), 95-120.

Vignal, M. (dir.) (1987 / 2005). Dictionnaire de la musique. Paris, France : Larousse. 